\title{
Progress towards agreement in reports of antibiotic sensitivity
}

\author{
R. H. GEORGE 1
}

From the Department of Bacteriology, The Birmingham Maternity Hospital, Edgbaston, Birmingham

SYNOPSIS Results of three quality control trials of antibiotic sensitivity testing carried out on staphylococci by the Birmingham Regional Bacteriologists Group are presented. An overall reduction in the number of discrepant results from 5.2 to $1.2 \%$ when retesting the same organisms was thought to be due largely to the alteration in methods of testing for resistance to neomycin- and penicillinase-resistant antibiotics. Improvements were noted in the performance of all laboratories, which were felt to be due partly to participation in the trials.

The results of at least five studies on the testing of antibiotic sensitivity by different laboratories have been published. In four of these trials laboratories carried out tests by their routine method. In two of these pure cultures were circulated (Institute of Medical Laboratory Technology, 1960; the College of Pathologists of Australia, 1968), and in another two clinical specimens were tested (Association of Clinical Pathologists, 1965; Stokes and Whitby, 1971). In yet another study by Beaney, Goodwin, Jones, Winter, and Sippe (1970) cultures were tested by a prescribed method. All these studies have shown erroneous reports of resistance and sensitivity.

Over the last two and a half years quality control trials have been carried out by the Birmingham Regional Bacteriologists Group. During this time four trials have been carried out on strains of staphylococci, two on enterobacteriaceae, and one on streptococci. This paper reports the results obtained with 10 isolates of staphylococci (nine coagulase positive) which were circulated on three separate occasions.

\section{Methods}

Cultures of staphylococci were allocated a different code letter in each trial and were sent by post on nutrient agar slopes to 16 laboratories. The mini-

'Other participants: G. A. J. Ayliffe, F. A. J. Bridgwater, P. P. Brown, D. W. Burdon, P. Cavanagh, I. Craig, M. M. Derrington, I. A. Harper, E. J. L. Lowbury, C. A. Morris, M. W. N. Nicholls, C. D. Plows, K. B. Rogers, M. B. Skirrow, J. E. M. Whitehead.

Received for publication 10 September 1974. mum inhibitory concentrations of penicillin, erythromycin, lincomycin, neomycin, and tetracycline were determined for each strain by an agar-dilution method at Dudley Road Hospital, Birmingham (table I).

Laboratories were asked to test sensitivity by their routine method. Two laboratories used the ditchplate technique with known resistant and sensitive strains on every plate, and the remaining laboratories used a disc-diffusion technique. Two of these used the Stokes method (1960), while the rest tested control organisms at intervals varying from daily to 'irregularly'. Of the laboratories testing sensitivity by the disc-diffusion technique all except two used commercially prepared sensitivity-test media and all except two standardized their inocula. A meeting of participants was held at the conclusion of each trial.

\section{Results}

A small number of reports of intermediate sensitivity were received from three laboratories. These were accepted as correct when analysing results unless the minimum inhibitory culture (MIC) had shown the organism to be highly sensitive or resistant. Disagreement occurred, however, with reports of sensitivity to neomycin on four strains with an MIC of $10 \mu \mathrm{g} / \mathrm{ml}$ as some members argued that these strains would respond to topical therapy. Because these strains show increased resistance they have been analysed as resistant strains with reports of sensitivity being regarded as 'discrepant'. The term 'discrepant result' is used throughout this report rather than erroneous, because of this difference with neomycin. 


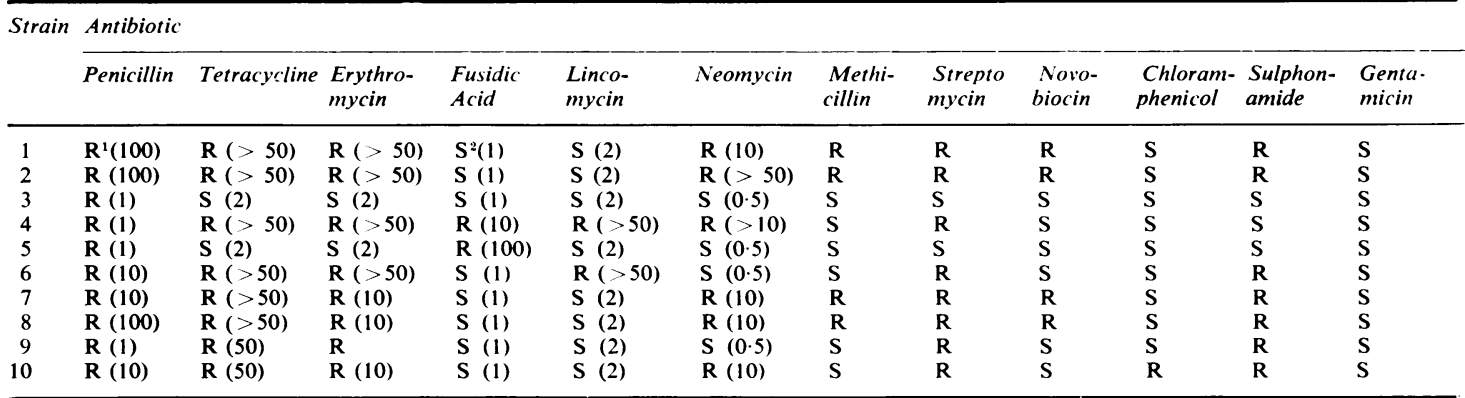

Table I Sensitivity pattern of strains of staphylococci ${ }^{1}$

${ }^{\mathbf{t}} \mathbf{R}=$ resistance

${ }^{2} \mathbf{S}=$ sensitive

Table II shows the number of tests and discrepant results for each antibiotic in these trials. Table III shows the percentage of discrepant reports of resistance when testing sensitive strains and table IV the percentage of discrepant reports of sensitivity when testing resistant strains.

There was a marked reduction in the number of discrepancies from an overall level of $5.2 \%$ in trial 1 to $1.2 \%$ in trial 3 . No problems were encountered when reporting gentamicin, novobiocin, rifampicin, or tetracycline, and very few discrepant reports occurred with chloramphenicol, erythromycin, fusidic acid, lincomycin, or penicillin. Penicillinase production was missed seven times in trial 1 (all from one laboratory, number 15) but was always detected in trials 2 and 3.

The overall reduction was mainly due to a reduction in the number of discrepancies when testing the penicillinase-resistant penicillins. It can be seen from table II that there was a change from testing cloxacillin to methicillin with a consequent reduction in the number of false reports of sensitivity. It seems likely that the reduction in the number of false reports of sensitivity to cephalosporins has a similar explanation, as many reports were by inference from tests with methicillin or cloxacillin. In trial 1 eight laboratories tested sensitivity to methicillin, five to cloxacillin, and one laboratory tested both. In trial 3 all laboratories tested sensitivity to methicillin only. However, one laboratory which had changec to testing methicillin sensitivity, used neither low temperature nor salt agar, with a number of disc crepancies resulting (table $\mathrm{V}$ ).

The problem of detecting staphylococci with an MIC of $10 \mu \mathrm{g} / \mathrm{ml}$ of neomycin was overcome partly due to an increase in the number of tests with kanamycin, and a small reduction in the testing of neomycin. In trials 2 and 3 there were, however, fewer

\begin{tabular}{|c|c|c|c|}
\hline \multirow[t]{2}{*}{ Antibiotic } & \multicolumn{3}{|l|}{ Discrepancies } \\
\hline & Trial 1 & Trial 2 & Trial 3 \\
\hline Penicillin & $7 / 149 \quad(4.7 \%)$ & $0 / 137 \quad(0 \%)$ & $0 / 139 \quad(0 \%)$ \\
\hline Ampicillin & $0 / 49 \quad(0 \%)$ & $0 / 49 \quad(0 \%)$ & $0 / 40 \quad(0 \%)$ \\
\hline Methicillin & $5 / 119 \quad(4.2 \%)$ & $5 / 130\left(3.9_{0}^{\circ}\right)$ & $3 / 139 \quad(2.2 \%)$ \\
\hline Cloxacillin & $14 / 33 \quad(42.4 \%)$ & $2 / 10(20.0 \%)$ & - - \\
\hline Cephalosporins & $15 / 50(30.0 \%)$ & $6 / 40\left(15 \cdot 0^{\circ}\right)$ & $0 / 30 \quad(0 \%)$ \\
\hline Gentamicin & $0 / 64 \quad(0 \%)$ & $0 / 83 \quad(0 \%)$ & $0 / 69 \quad(0 \%)$ \\
\hline Neomycin & $17 / 69 \quad(24.6 \%)$ & $5 / 79 \quad(6.3 \%)$ & $3 / 50 \quad(6.0 \%)$ \\
\hline Kanamycin & $1 / 29 \quad(3.4 \%)$ & $0 / 50 \quad(0 \%)$ & $0 / 69 \quad(0 \%)$ \\
\hline Streptomycin & $5 / 69 \quad(7 \cdot 2 \%)$ & $2 / 60 \quad(3.3 \%)$ & $3 / 49 \quad(6.1 \%)$ \\
\hline Lincomycins & $1 / 147 \quad(0.7 \%)$ & $1 / 120(0.8 \%)$ & $4 / 139(2.9 \%)$ \\
\hline Fusidic acid & $2 / 138 \quad(1.4 \%)$ & $3 / 131 \quad(2 \cdot 3 \%)$ & $1 / 139(0.7 \%)$ \\
\hline Erythromycin & $0 / 124(0 \%)$ & $1 / 122(0.8 \%)$ & $1 / 129(0.8 \%)$ \\
\hline Chloramphenicol & $1 / 49 \quad(2.0 \%)$ & $1 / 50 \quad(2.0 \%)$ & $0 / 59 \quad(0 \%)$ \\
\hline Tetracycline & $0 / 149(0 \%)$ & $0 / 138(0 \%)$ & $0 / 139(0 \%)$ \\
\hline Novobiocin & $0 / 45 \quad(0 \%)$ & $0 / 42 \quad(0 \%)$ & $0 / 39(0 \%)$ \\
\hline Rifampicin & $0 / 30 \quad(0 \%)$ & $0 / 20 \quad(0 \%)$ & $0 / 29 \quad(0 \%)$ \\
\hline Cotrimoxazole & $4 / 53 \quad(7.5 \%)$ & $0 / 102 \quad(0 \%)$ & $0 / 89 \quad(0 \%)$ \\
\hline Sulphonamides & $4 / 30(13.3 \%)$ & $5 / 50 \quad(10.0 \%)$ & $3 / 59 \quad(5.1 \%)$ \\
\hline Others & $0 / 50 \quad(0 \%)$ & $0 / 40 \quad(0 \%)$ & $0 / 39 \quad(0 \%)$ \\
\hline Total & $76 / 1446(5 \cdot 2 \%)$ & $31 / 1453(2.1 \%)$ & $18 / 1445(1.2 \%)$ \\
\hline
\end{tabular}

Table II Discrepant reports on staphylococci 


\begin{tabular}{lccc}
\hline Antibiotic & \multicolumn{2}{l}{ Trial } & \\
\cline { 2 - 4 } & 1 & 2 & 3 \\
\hline Penicillin & -2 & - & - \\
Ampicillin & - & - & - \\
Methicillin & 0 & $2 \cdot 6(2)$ & 0 \\
Cloxacillin & 0 & 0 & - \\
Cephalosporins & 0 & 0 & 0 \\
Gentamicin & 0 & 0 & 0 \\
Neomycin & $2 \cdot 9(1)$ & 0 & 0 \\
Kanamycin & 0 & 0 & 0 \\
Streptomycin & $4 \cdot 8(1)$ & 0 & $15 \cdot 0(3)$ \\
Lincomycins & $0 \cdot 8(1)$ & 0 & $2 \cdot 7(3)$ \\
Fusidic acid & $0 \cdot 9(1)$ & $1 \cdot 9(2)$ & $0 \cdot 9(1)$ \\
Erythromycin & 0 & 0 & 0 \\
Chloramphenicol & 0 & $2 \cdot 0(1)$ & 0 \\
Tetracycline & 0 & 0 & 0 \\
Novobiocin & 0 & 0 & 0 \\
Rifampicin & 0 & 0 & 0 \\
Cotrimoxazole & $7 \cdot 5(4)$ & 0 & 0 \\
Sulphonamides & $44 \cdot 4(4)$ & $10 \cdot 0(2)$ & $17 \cdot 7(3)$ \\
Others & 0 & 0 & 0 \\
Total & $1 \cdot 7$ & $0 \cdot 9$ & $1 \cdot 3$ \\
\hline
\end{tabular}

Table III Discrepant reports of resistance ${ }^{1}$

${ }^{1}$ Expressed as percentage of tests on sensitive organisms with actual number of discrepancies in parentheses.

2 - = no sensitive organisms tested

\begin{tabular}{lccc}
\hline Antibiotic & \multicolumn{2}{l}{ Trial } & \\
\cline { 2 - 4 } & 1 & 2 & 3 \\
\hline Penicillin & $4 \cdot 7(7)$ & 0 & 0 \\
Ampicillin & 0 & 0 & 0 \\
Methicillin & $10 \cdot 4(5)$ & $5 \cdot 4(3)$ & $5 \cdot 4(3)$ \\
Cloxacillin & $82 \cdot 4(14)$ & $50 \cdot 0(2)$ & - \\
Cephalosporins & $60 \cdot 0(15)$ & $50 \cdot 0(2)$ & 0 \\
Gentamicin & -2 & - & - \\
Neomycin & $470(16)$ & $16 \cdot 1(5)$ & $12 \cdot 0(3)$ \\
Kanamycin & $7 \cdot 1(1)$ & 0 & 0 \\
Streptomycin & $8 \cdot 3(4)$ & $8 \cdot 3(2)$ & 0 \\
Lincomycins & 0 & $4 \cdot 3(1)$ & $3 \cdot 6(1)$ \\
Fusidic acid & $3 \cdot 8(1)$ & $4 \cdot 0(1)$ & $3 \cdot 6(1)$ \\
Erythromycin & 0 & 0 & $1 \cdot 0(1)$ \\
Chloramphenicol & $20 \cdot 0(1)$ & 0 & 0 \\
Tetracycline & 0 & 0 & 0 \\
Novobiocin & 0 & 0 & 0 \\
Rifampicin & - & - & - \\
Cotrimoxazole & - & - & - \\
Sulphonamides & 0 & $10 \cdot 0(3)$ & 0 \\
Others & 0 & 0 & 0 \\
Total & $8 \cdot 8$ & $3 \cdot 8$ & $1 \cdot 1$ \\
\hline
\end{tabular}

Table IV Discrepant reports of sensitivity ${ }^{1}$

'Expressed as percentage of tests on sensitive organisms with actual number of discreprancies in parenthesis.

$z_{-}=$no resistant organisms tested

discrepant reports of sensitivity in those testing neomycin, which must have been due to better interpretation of zones in relation to controls.

One laboratory, number 7 , which performed predominantly direct sensitivity tests on blood agar no. 2 (Oxoid) with added lysed and whole blood had difficulty testing sulphonamides throughout the trials. This may be related to the inoculum size as much as to composition of the medium. However,

\begin{tabular}{llll}
\hline Medium & $\begin{array}{c}\text { Temperature } \\
\left({ }^{\circ} \mathrm{C}\right)\end{array}$ & $\begin{array}{l}\text { No. of Tests } \\
\text { on Resistant } \\
\text { Organisms }\end{array}$ & $\begin{array}{l}\text { No. of } \\
\text { Discrepancies }\end{array}$ \\
\hline $\begin{array}{l}5 \% \text { salt agar } \\
5 \% \text { salt agar }\end{array}$ & 30 & 28 & $0(0 \%)$ \\
$\begin{array}{l}\text { Wellcotest agar } \\
\text { (Wellcome Reagents }\end{array}$ & 37 & 67 & $1(1 \cdot 5 \%)$ \\
$\quad$ Ltd) & 30 & 4 & $0(0 \%)$ \\
$\begin{array}{l}\text { DST agar (Oxoid) } \\
\text { DST agar (Oxoid) }\end{array}$ & 30 & 30 & $4(13 \cdot 3 \%)$ \\
$\begin{array}{l}\text { New Zealand agar } \\
\text { (ditch test) }\end{array}$ & 30 & 4 & $3(75 \cdot 0 \%)$ \\
\hline
\end{tabular}

Table V Method of testing methicillin sensitivity

the level of performance for each laboratory without exception improved (table VI) in later trials.

The number of antibiotics reported by different laboratories ranged from six to 17 with a mean of 9.6 in trial 1 , and means of 10.3 in both subsequent trials.

\begin{tabular}{|c|c|c|c|c|}
\hline \multirow[t]{2}{*}{ Laboratory } & \multirow[t]{2}{*}{ Method } & \multicolumn{3}{|l|}{ Trial } \\
\hline & & 1 & 2 & 3 \\
\hline 1 & Disc diffusion & 6.2 & 0 & $1 \cdot 1$ \\
\hline 2 & Stokes & $2 \cdot 5$ & $2 \cdot 0$ & -1 \\
\hline 3 & Ditch plate & 6.0 & $6 \cdot 2$ & 0.9 \\
\hline 4 & Disc diffusion & 0 & 0 & - \\
\hline 5 & Stokes & $4 \cdot 4$ & 0.9 & 0.9 \\
\hline 6 & Disc diffusion & $1 \cdot 3$ & $1 \cdot 1$ & 0.9 \\
\hline 7 & Disc diffusion & 8.5 & $4 \cdot 3$ & $4 \cdot 3$ \\
\hline 8 & Disc diffusion & 1.0 & - & 0 \\
\hline 9 & Disc diffusion & $8 \cdot 2$ & 0 & 0.9 \\
\hline 10 & Disc diffusion & 3.0 & 1.8 & 0 \\
\hline 11 & Disc diffusion & 5.0 & $1 \cdot 1$ & 0 \\
\hline 12 & Disc diffusion & $7 \cdot 1$ & $5 \cdot 7$ & $\mathbf{0}$ \\
\hline 13 & Disc diffusion & 8.7 & - & $6 \cdot 7$ \\
\hline 14 & Disc diffusion & - & $4 \cdot 4$ & $2 \cdot 5$ \\
\hline 15 & Ditch plate & $11 \cdot 6$ & $2 \cdot 5$ & $\mathbf{0}$ \\
\hline 16 & Disc diffusion & 0.7 & 0.7 & 0 \\
\hline
\end{tabular}

Table VI Discrepancies as percentage of reports from each laboratory

$1-=$ no results received

\section{Discussion}

The object of quality control trials is to improve the performance of laboratories when testing antibiotic sensitivity. The advantages of trials involving a small number of participants who met regularly and discussed results was felt to play a part in the reduction of faults by Stokes and Whitby (1971), although they felt that their numbers were too small to draw definite conclusions. Similar influences were probably important in the present trials, as changes in the method of testing resistance to a small number of antibiotics was the major factor in the reduction of discrepant reports. Although our laboratories knew 
that the same strains had previously been circulated, we do not feel this was a significant factor in their improvement.

The number of antibiotics reported by laboratories during these trials varied from six in one laboratory to 17 in another. Many laboratories, however, tested sensitivity to both first and second 'line' antibiotics only because they were participating in our trials.

It is interesting to note that the overall level of performance even in trial 1 was higher than that found by the College of Pathologists of Australia (1968). This probably reflects the greater awareness today of the problems involved, the most striking example being the detection of penicillinase-producing staphylococci. All the false reports of sensitivity to penicillin were from one laboratory in trial 1 using the ditch-plate technique. Subsequent investigation revealed a penicillin concentration within the ditch one hundred-fold higher than normally used.

The frequency with which sensitivity tests to cloxacillin were performed in trial 1 is perhaps surprising in view of reports by Hewitt, Coe, and Parker (1969) and Garrod and Waterworth (1971) of their unreliability. Castle and Elstub (1971) in a survey of 330 laboratories in the United Kingdom found that 162 were testing sensitivity to cloxacillin and 84 to methicillin in first-line tests. Our findings suggest that a considerable number of laboratories may still be performing these tests. All our laboratories have changed to testing methicillin as a direct result of participation in these trials.

The failure to detect moderate resistance to neomycin in early trials is difficult to explain, as reduced zones should have been noted by laboratories using adequate control organisms. The substitution of testing kanamycin instead of neomycin reduced this problem. This was previously reported by Waterworth (1969).

The use of standard methods to improve the quality of antibiotic sensitivity tests has been proposed by Bauer, Perry, and Kirby (1959), by Bauer, Kirby, Sherris, and Turck (1966), and by Ericsson and Sherris (1971). Beaney et al (1970) used standard methods throughout their quality control trials and still demonstrated a considerable number of erroneous reports, suggesting either faulty execution of the methods or problems in interpretation. In the present trials no attempt was made to standardize the techniques for performing tests. The variation in antibiotic content of discs used by different laboratories was not found to be a reason for discrepant results. Our results suggest, therefore, that for staphylococci it may not be necessary to standardize beyond a certain point. Garrod and Waterworth (1971) have made proposals for simple methods to improve reliability of reporting. The situation is clearly different in the case of enterobacteriaceae when our trials have shown that variation in the antibiotic content of discs is important.

In conclusion, our members feel that participation in such a scheme has led to an improvement in the performance of antibiotic sensitivity tests. It is open to debate whether further improvement would accrue from extending the trials on staphylococci. For the time being we have felt it more valuable to carry out trials on enterobacteriaceae and other bacteria.

I am grateful to Dr G. A. J. Ayliffe and Dr J. D. Williams for providing the strains and organizing the survey.

\section{References}

Association of Clinical Pathologists (1965). Report on antibiot sensitivity test trial organised by the Bacteriology Committe of the Association of Clinical Pathologists. J. clin. Path., 18त् $1-5$.

Bauer, A. W., Kirby, W. M. M., Sherris, J. C., and Turck, M. (1966?. Antibiotic susceptibility testing by a standardised single-disk method. Amer. J. clin. Path., 45, 493-496.

Bauer, A. W., Perry, D. M., and Kirby, W. M. M. (1959). Single-disk antibiotic-sensitivity testing of staphylococci. Arch. intern. Med., 104, 208-216.

Beaney, N., Goodwin, N. G., Jones, R. V., Winter, R., and Sippe, G. R. (1970). Antibiotic sensitivity testing. Med. J. Aust., 1, 483-484.

Castle, A. R., and Elstub, J. (1971). Antibiotic sensitivity testing: a survey undertaken in September 1970 in the United Kingdom. J. clin. Path., 24, 773-778.

College of Pathologists of Australia (1968). A survey of antibiotic sensitivity testing. Med. J. Aust., 2, 171-172.

Ericsson, H. M., and Sherris, J. C. (1971). Antibiotic sensitivity testing report of an international collaborative study. Actí path. microbiol. scand., Sect. B., Suppl. 217.

Garrod, L. P., and Waterworth, P. M. (1971). A study of antibiotic sensitivity testing with proposals for simple uniform methods. J. clin. Path., 24, 779-789.

Hewitt, J. H., Coe, A. W., and Parker, M. T. (1969). The detection of methicillin resistance in Staphylococcus aureus. J. med. Micro biol, 2, 443-456.

Institute of Medical Laboratory Technology (1960). A survey of antibiotic sensitivity tests. J. med. Lab. Technol., 17, 133-143.

Stokes, E. J. (1968). Clinical Bacteriology, 3rd ed., p. 179. Arnold, హ) London.

Stolies, E. J., and Whitby, J. L. (1971). Quality control in bacterıology: preliminary trials. J. clin. Path., 24, 790-797.

Waterworth, P. M. (1969). Interpretation of disc sensitivity tests on organisms of intermediate sensitivity. J. med. Lab. Technol., 26, 106-110. 